Service social

\title{
Intervention de groupe auprès des personnes atteintes de brûlures
}

\section{Aline Bolduc}

Volume 45, numéro 3, 1996

Santé

URI : https://id.erudit.org/iderudit/706742ar

DOI : https://doi.org/10.7202/706742ar

Aller au sommaire du numéro

\section{Éditeur(s)}

École de service social de l'Université Laval

ISSN

1708-1734 (numérique)

Découvrir la revue

Citer cet article

Bolduc, A. (1996). Intervention de groupe auprès des personnes atteintes de brûlures. Service social, 45(3), 137-152. https://doi.org/10.7202/706742ar

\section{Résumé de l'article}

Les personnes atteintes de brûlures vivent un traumatisme qui se double d'une image corporelle modifiée et de risques de séquelles graves. Elles auront toujours besoin du soutien d'autrui, et celui apporté par la famille se révèle le plus important dans le processus de leur réadaptation. Devant cette situation, les proches se sentent démunis et éprouvent un sentiment d'impuissance et de culpabilité. Ils ont besoin d'assistance pour être capables de bien vivre cette expérience. Cet article présente la problématique des personnes atteintes de brûlures et l'intervention psychosociale telle que proposée par l'Association des grands brûlés FLAM à ses membres. FLAM constitue un organisme communautaire dont le but est spécifiquement d'œuvrer auprès de la clientèle des personnes qui ont été gravement brûlées. 


\section{Intervention de groupe auprès des personnes atteintes de brûlures}

Aline BOLDUC Étudiante à la maîtrise en service social Intervenante sociale Association des grands brûlés FLAM

Dans la vie quotidienne, bien des personnes doivent vivre des événements stressants, pénibles. Subir un accident par brûlures fait partie de ces situations très difficiles auxquelles certains doivent faire face. De fait, les personnes atteintes de brûlures vivent un traumatisme avec des altérations permanentes de leur apparence et, dans certains cas, des séquelles physiques, psychologiques et sociales importantes. Certaines demeurent même gravement handicapées sur le plan fonctionnel. À la suite d'un accident causant des brûlures, une longue période de réadaptation physique et psychologique s'impose habituellement.

Pendant cette période, les personnes atteintes de brûlures ont besoin du soutien de leur milieu familial et de leur environnement social et professionnel pour les aider à traverser cette épreuve et retrouver un niveau de fonctionnement adéquat. 
Mon rôle, en tant qu'intervenante sociale œuvrant auprès de l'Association des grands brûlés FLAM (Force, Liberté, Amour et Mouvement d'accueil aux victimes de brûlures), consiste à aider ces personnes à faire face aux difficultés qu'elles éprouvent et à apporter soutien et réconfort à leur entourage. FLAM propose aux personnes atteintes de brûlures et à leurs proches un programme comprenant une intervention individuelle et une intervention de groupe. L'intervention individuelle s'adresse à la personne qui exprime des besoins particuliers. Quant à l'intervention de groupe, elle poursuit des objectifs d'entraide des individus et des familles. Comme l'intervention de groupe occupe une place dominante dans le programme offert, nous ne ferons mention ici que de cette forme de soutien.

L'objet du présent article est donc de faire connaître la problématique des personnes atteintes de brûlures et de présenter l'intervention de groupe menée auprès de cette clientèle.

\section{LA PROBLÉMATIQUE DES PERSONNES ATTEINTES DE BRÛLURES}

L'association américaine des grands brûlés définit un grand brûlé comme une personne qui a subi des brûlures aux $2^{\mathrm{e}}$ et $3^{\mathrm{e}}$ degrés et dont $20 \%$ ou plus de la surface corporelle est atteinte. Ce critère est plus bas lorsqu'il s'agit d'enfants et de personnes âgées, leur peau étant plus délicate et l'immunité plus faible (Weinberg et Miller, 1983, p. 98). Par contre, le Dr Henri-Louis Bouchard, cofondateur de l'association des grands brûlés ici, définit le grand brûlé comme une personne qui peut être victime d'une brûlure peu étendue en ce qui a trait à la surface corporelle, mais dont les séquelles sont perçues de façon disproportionnée à leur réalité. Il ajoute que ce n'est ni l'étendue ni le degré de brûlure qui devraient définir le grand brûlé, mais le traumatisme psychologique lié à la perception qu'a la victime au regard des pertes sur le plan personnel, professionnel et social.

\section{Caractéristiques des grands brûlés}

Les blessures par brûlures affectent les gens de tous les âges. D'après les données disponibles, le groupe des enfants âgés de un à quatre ans est celui où l'on compte le plus grand nombre de victimes de brûlures pour la période 1985-1993. Ce groupe 
représente environ $18 \%$ de toute la population atteinte de brûlures. Viennent ensuite, par ordre décroissant, les adultes âgés de 25 à 34 et ceux âgés de 45 à 64 ans. La population masculine semble plus susceptible de subir des blessures par brûlures. En fait, $70 \%$ des personnes hospitalisées sont des hommes (ministère de la Santé nationale et du Bien-être social, 1985, p. 3 ; Statistique Canada, Morbidité hospitalière). Cette situation s'expliquerait par le fait que les hommes occupent probablement plus que les femmes des emplois à haut risque de brûlures, en exécutant par exemple des travaux qui nécessitent l'utilisation plus fréquente de l'électricité, du feu et de produits chimiques ou explosifs (Munster, 1993, p. XVII).

La résidence familiale est généralement l'endroit où les accidents par brûlures surviennent (66\%) (Weinberg et Miller, 1983). La cuisine est la pièce où se produit le plus souvent ce genre d'accident. Quant aux agents responsables de ces accidents, les plus souvent mentionnés sont le feu (47\%), l'eau bouillante (16\%) et l'électricité (13\%) (Munster, 1993, p. XVIII). $\mathrm{Au}$ cours des dernières années, les cas de brûlures attribuables aux accidents de travail, à la manipulation de produits dangereux et aux incendies ont diminué. En effet, le Centre suprarégional de l'hôpital Saint-Sacrement a reçu plus fréquemment des personnes âgées en perte d'autonomie qui ont mis le feu à leurs vêtements en cuisinant, des individus qui ont consommé des drogues ou de l'alcool et qui se sont endormis en fumant, des enfants qui ont subi des brûlures infligées par leurs parents et, plus rarement, des cas d'immolation. En certaines occasions, des personnes ayant des problèmes de santé mentale provoquent l'incendie de leur demeure (Gagnon, 1994).

L'étude des caractéristiques des personnes victimes de brûlures fait ressortir que des conditions pathologiques, telles qu'une maladie dégénérative, un retard mental, l'obésité, la sénilité, peuvent accroître le risque de brûlures. Il en est de même de la consommation abusive d'alcool ou de drogue et de la présence de troubles émotionnels ou psychiatriques. Enfin, des facteurs sociaux tels l'ignorance, l'imprudence et le niveau de vie économiquement bas seraient liés aux blessures par brûlures (Gardney, 1981, p. 136). Chez les enfants, des troubles de comportement seraient associés à une certaine propension à subir des brûlures (Weinberg et Miller, 1983, p. 97). 


\section{La réadaptation}

La sortie de l'hôpital constitue un moment charnière dans la démarche de réadaptation de la personne victime de brûlures. Celle-ci doit affronter ses limites physiques et psychologiques et lutter pour retrouver son autonomie. Les activités de la vie quotidienne les plus simples, telles que s'alimenter, se mouvoir, s'asseoir, se lever, se pencher, s'étirer et procéder à ses soins d'hygiène personnelle, peuvent être très difficiles à exécuter. Les suites du traumatisme entraînent des changements de l'apparence physique et comportent des risques de séquelles permanentes (Villeneuve, 1987). La personne qui a subi des brûlures doit faire le deuil de son image corporelle, que ce soit en raison de cicatrices permanentes, de perte d'amplitude de certaines articulations ou même d'amputation. Enfin, plusieurs doivent s'astreindre à porter des vêtements compressifs pour favoriser la guérison et diminuer les démangeaisons au niveau des sites donneurs (zones épargnées par l'accident où sont prélevées des sections d'épiderme sain, c'est-à-dire des greffons) et des sites greffés (zones brûlées, débridées de leurs tissus nécrosés et recouvertes de peau saine).

Comme la sortie de l'hôpital marque le début d'une longue période de réadaptation, c'est une étape déterminante pour l'évolution future de la personne (Noël, 1991). Souvent, des interventions chirurgicales correctrices doivent être planifiées. De nombreux ajustements s'imposent pour que la personne brûlée remplisse à nouveau ses rôles familiaux, sociaux et professionnels (Weinberg et Miller, 1983). L'accident par brûlures est donc un accident de courte durée, souvent suivi de plusieurs années de souffrance pour la victime ainsi que pour ses proches qui l'accompagnent dans son cheminement (Mannon, 1985). Par ailleurs, la personne convalescente doute souvent de l'habileté des siens à lui assurer des soins physiques de qualité et à lui prodiguer les conseils appropriés à son état psychologique.

Le problème esthétique constitue le handicap le plus difficile à surmonter pour la personne atteinte de brûlures et porteuse de cicatrices. Lorsqu'elle se regarde dans le miroir, elle ne reconnaît pas l'image qui s'y reflète. C'est une image corporelle altérée, difficile à regarder et à accepter. Marquée dans sa chair à tout jamais, la personne doit passer par une période d'adaptation qui n'est pas sans lui causer de graves problèmes psychologiques. La personne victime de brûlures ressent une atteinte profonde de son moi et éprouve de grandes difficultés à vaincre 
son angoisse devant ses cicatrices et son aspect visuel. La perte de l'image de soi peut parfois précipiter la personne dans un état dépressif (Knudson-Cooper, 1981).

L'altération de l'image corporelle provoque chez la personne victime de brûlures un sentiment de dépréciation personnelle. Elle refuse de s'aimer. Elle ira même jusqu'à s'interdire le droit d'aimer et d'être aimée. Certains brûlés n'osent plus avoir de relations sexuelles avec leur conjoint, pensant qu'ils ne sont plus attrayants physiquement. Le changement de leur apparence peut les amener à ressentir de la honte, de la crainte ou d'autres émotions négatives qui nuisent à leur intimité. Ils craignent d'être rejetés. Ils peuvent se sentir moins aptes à la poursuite de leur relation amoureuse. Il arrive même qu'ils se cachent devant leur partenaire ou acceptent d'une façon passive ses avances (Munster, 1993, p. 103).

Il est reconnu que notre société moderne base, la plupart du temps, la valeur des gens sur des critères esthétiques définis et des règles préétablies. Elle peut donc devenir cruelle à l'égard des personnes présentant un physique atypique et dont l'apparence provoque un recul instinctif ou de l'antipathie. Les personnes victimes de brûlures sont souvent l'objet de regards malveillants et de questions indiscrètes. Ces situations deviennent des épreuves difficiles à surmonter.

La période d'incapacité des personnes victimes de brûlures est toujours liée au caractère et à l'importance des séquelles. Le retour au travail et à la vie professionnelle devient parfois complexe et fait suite à une absence d'une durée variable (Weinberg et Miller, 1983). Un certain nombre de victimes se recyclent dans un autre domaine, parce qu'un handicap fonctionnel les empêche d'exécuter le travail fait auparavant. Des victimes sont, malheureusement, tout simplement remerciées de leurs services, leurs supérieurs leur signifiant que leur poste est aboli. Parfois, se sentant dépréciée par son employeur, une personne victime de brûlures choisit de démissionner. Les victimes de brûlures peuvent consacrer un an, deux ans de leur vie, parfois plus, à se rétablir physiquement. Dans d'autres cas, toute reprise d'activité professionnelle est exclue, et la personne est déclarée invalide (Quattrina, 1993).

Au cours de la période de réinsertion sociale, il est fréquent de constater que la personne fait souvent référence à son état antérieur, à son passé professionnel, social et familial, à ce qu'elle n'a plus (Noël, 1991). En fait, à ce qu'elle n'est plus. Car, 
d'une part, l'être humain se définit à travers ses activités et, d'autre part, la société reconnaît et valorise, jusqu'à un certain point, la personne au regard des activités qu'elle fait. Pour la plupart des gens, les activités de travail constituent la référence à travers laquelle ils se définissent et sont définis par autrui (Munster, 1993). Pendant un temps indéterminé, certains sont empêchés de remplir le ou les rôles dévolus par leur statut familial et social, tel le rôle de pourvoyeur. Car un certain nombre de victimes sont parfois plusieurs années dans l'incapacité de travailler. Elles ne peuvent donc plus se définir en fonction de leurs tâches antérieures et elles ne se reconnaissent plus. Pour les unes, c'est la catastrophe financière, car elles ne reçoivent pas d'indemnité. De sorte que les personnes atteintes de brûlures sont, à certains moments, déstabilisées.

Plusieurs personnes porteuses de cicatrices cessent de pratiquer leurs sports favoris et s'abstiennent de fréquenter les lieux publics, tels les restaurants, les centres commerciaux et les transports publics, de peur d'être un sujet de curiosité. Elles s'interrogent sur les réactions des autres concernant leur apparence. Étant donné qu'elles sont constamment préoccupées par leur image corporelle modifiée et abîmée, elles supposent que toutes les personnes y portent cette même attention (Villeneuve, 1987, p. 56). Certaines vont jusqu'à craindre qu'on découvre leurs cicatrices dissimulées par les vêtements et elles ont tendance à s'isoler.

De plus, ces personnes sont parfois délaissées par leurs amis et pressentent douloureusement la pitié, la désolation et les moqueries que leur apparence provoque (Mannon, 1985). Les rapports avec leur famille, leurs voisins et la société en général peuvent leur sembler étranges et devenir inconfortables. Les personnes victimes de brûlures doivent combattre quotidiennement pour retrouver le goût de vivre, l'amour de soi et l'acceptation de la société qui les entoure (Noël, 1991).

\section{Les besoins des personnes victimes de brûlures}

Les personnes victimes de brûlures se retrouvent dans une situation nouvelle. Aimer et être aimé devient un besoin prioritaire. C'est la condition essentielle pour établir de nouveaux liens d'amitié. Une autobiographie réalisée par une personne victime de brûlures, intitulée Aimez-moi, illustre bien ce grand besoin (Giguère, 1988). Ce thème, l'auteure l'a maintes fois clamé sous 
diverses formes. Il est demeuré, pour elle, ce désir fondamental et cette lueur d'espoir qui l'ont préservée de la mort et de la solitude. Les personnes victimes de brûlures éprouvent un immense besoin de contact humain, d'amitié et d'attention (Burg, 1991, p. 27). Elles veulent être traitées avec bienveillance et ne pas être perçues comme un objet de curiosité. Ainsi, elles pourront reprendre confiance en elles et s'accepter plus facilement.

De plus, les personnes victimes de brûlures auront toujours besoin du soutien familial, du soutien d'autrui ou d'aide psychologique (Villeneuve, 1987, p. 57). Le soutien apporté par la famille se révèle le plus important dans le processus de leur réadaptation. C'est un rôle capital qui consiste à encourager continuellement les personnes atteintes de brûlures dans le but de leur faire recouvrer leur autonomie. Les efforts que ces dernières font en ce sens s'en trouvent renforcés. C'est pourquoi le moindre progrès réalisé dans les activités courantes et dans les exercices prescrits doit être souligné (Munster, 1993). Bien que, habituellement, les personnes atteintes de brûlures tardent à demander de l'aide, elles ressentent le besoin de partager leur vécu quotidien, de verbaliser la colère et les autres émotions, de parler de leurs difficultés.

La personne victime de brûlures vivait normalement au milieu d'une famille, d'amis, de collègues d'étude ou de travail avant l'accident. Cette perturbation, qu'elle soit temporaire ou permanente, bouleverse tous ces gens. Il leur est difficile d'accepter le fait qu'une blessure par brûlures ait changé irrémédiablement une personne de leur entourage (Munster, 1993). Ils sont pris de panique devant cette situation tout à fait inattendue. Ils se sentent démunis et éprouvent un sentiment d'impuissance devant une réalité qui leur semble sans recours. Ils se sentent coupables et responsables des circonstances de l'accident. En plus de s'inquiéter de la survie physique de la personne victime de brûlures, ils peuvent se faire du souci au point de vue de la sécurité financière, du travail, des enfants et de la maison. Ils ressentent de l'angoisse relativement à l'avenir incertain de l'être aimé.

L'image de la brûlure et des cicatrices, l'incapacité, même temporaire, de la personne atteinte de brûlures à remplir ses rôles ainsi que les attitudes néfastes de la population les affectent autant que la personne atteinte de brûlures (Munster, 1993). 
Par contre, certains ont tendance à surprotéger les victimes de brûlures. Si la surprotection est satisfaisante pour ceux qui entourent la victime de brûlures, elle est souvent plus nuisible qu'efficace pour cette dernière, parce qu'elle retarde la prise de contact avec la réalité (Noël, 1991, p. 27).

Les proches se trouvent désemparés, ne possédant aucune notion de la problématique de la personne victime de brûlures. Ils ont besoin d'information sur les conseils appropriés à donner aux réactions de la victime, sur les soins médicaux prescrits ainsi que sur les ressources disponibles dans le réseau de la santé. Ils ont également besoin d'assistance pour être capables de traverser cette épreuve. Bien souvent, ils doivent s'improviser infirmier ou infirmière et se tenir à l'écoute des doléances d'une personne frêle et vulnérable qui a grand besoin de réconfort. Lorsque la personne victime de brûlures vit en couple, le conjoint doit parfois recourir à une aide concrète pour exécuter diverses tâches.

En fait, chacune de ces personnes, tant les victimes de brûlures que leurs proches, a besoin de se raconter et d'être écoutée. Le fait qu'elle s'exprime facilite une sorte de décharge émotionnelle qui lui permet d'avoir une perception plus claire, plus objective d'elle-même et de la situation dans laquelle elle se trouve.

Pour faire face à cette réalité nouvelle, les personnes victimes de brûlures ont besoin du soutien des membres de leur famille et de leurs amis. Le soutien social est considéré comme un facteur significatif qui réduit la détresse psychologique éprouvée par les personnes qui vivent des événements stressants (Renaud et Tessier, 1993, p. 8-9). Le soutien émotionnel, le soutien informationnel, l'aide concrète, le soutien de l'estime de soi et l'intégration sociale constituent les différents types du soutien apporté par les proches (Cutrona et Russell, 1990, dans Turcotte, 1993, p. 95). Les personnes qui donnent du soutien peuvent être des personnes significatives du réseau personnel des victimes de brûlures. Ce peut être aussi des personnes qui, par leur expérience de vie et leurs caractéristiques personnelles, deviennent des sources significatives de soutien.

Les transformations sociales récentes provoquent parfois un effritement du réseau de soutien social, d'où la nécessité d'élaborer une intervention pour créer une structure de soutien. 


\section{L'INTERVENTION DE GROUPE}

L'aide mutuelle, comme fondement de l'intervention de groupe, peut s'avérer un moyen de prédilection approprié pour répondre aux besoins de soutien psychologique et affectif des personnes en difficulté. En s'associant avec des personnes qui font face à des problèmes semblables, ces personnes se sentent moins isolées dans leurs épreuves et libèrent plus facilement leur culpabilité. L'aide mutuelle les rend plus conscientes de la force personnelle dont elles disposent pour surmonter leurs difficultés. Cette aide est définie comme étant «le mécanisme par lequel les membres se supportent pour travailler à des tâches communes» (Home et Darveau-Fournier, 1980, p. 21).

L'aide mutuelle est un processus de groupe qui ne se manifeste pas automatiquement lorsque les personnes se rassemblent. Certaines conditions en facilitent l'émergence. Partager des informations sur les difficultés et les solutions constitue le premier processus d'aide mutuelle. Échanger des opinions ou des perceptions est une occasion privilégiée d'apprentissage et de développement personnel à cet égard. L'acceptation et l'attention chaleureuse du groupe sont de précieuses sources psychologiques et affectives pour chacun des membres (Shulman, 1979).

Ce sont les connaissances acquises par l'expérience qui permettent aux membres de fournir de l'aide et de susciter des réflexions et des échanges pertinents (Hopmeyer, 1990, p. 64). Les membres deviennent peu à peu des ressources les uns pour les autres. Ils utilisent leurs propres expériences pour aider l'autre à comprendre les différents aspects de son problème, à considérer celui-ci sous un angle différent et à trouver éventuellement des solutions (Shulman, 1979). Cette démarche de collaboration et de coopération introduit des rapports égalitaires entre les membres du groupe. Dans cette perspective, l'intervention de groupe prend appui sur le modèle de réciprocité dont la pierre angulaire est l'entraide (Papell et Rothman, 1966, p. 74).

Au début des années 1990, l'Association des grands brûlés FLAM a mis sur pied un groupe de soutien. Cette association est un organisme de charité enregistré. Elle a été fondée, en mars 1985, à la demande d'une victime de brûlures de la région de Québec et de sympathisants à sa cause. Cet organisme a pour mission d'améliorer la qualité de vie des victimes de brûlures et de leurs proches pendant l'hospitalisation, après la sortie de 
l'hôpital et au cours de la période de réadaptation. Les objectifs associés à cette mission sont:

- Regrouper en association les victimes de brûlures.

- Favoriser leur autonomie.

- Faciliter leur réinsertion dans la société.

- Promouvoir et défendre leurs intérêts.

- Créer des services pertinents à leurs besoins et à leurs attentes.

Cet organisme communautaire offre des services de soutien aux personnes victimes de brûlures du territoire de l'Est-duQuébec ainsi qu'à leurs proches. Il regroupe près de 250 membres, dont $27 \%$ sont des personnes atteintes de brûlures, $25 \%$ des proches et $48 \%$ des sympathisants.

Le groupe de soutien offert peut être défini comme un petit groupe formé de personnes affectées par les brûlures, qui se réunissent dans le but de s'aider mutuellement. L'entraide suppose que la personne désireuse de se joindre au groupe est motivée à s'aider elle-même tout en acceptant le soutien du groupe. Elle est principalement vécue lors des rencontres mensuelles. Le soutien informationnel et l'intégration sociale constituent les types de soutien les plus souvent manifestés. Le soutien informationnel concerne le partage d'information liée à la problématique des personnes affectées par les brûlures, alors que l'intégration sociale renvoie au sentiment de faire partie d'un groupe qui partage des difficultés communes.

L'Association des grands brûlés FLAM croit que les personnes victimes de brûlures et leurs proches possèdent des capacités et des habiletés pour s'engager et pour participer à des activités. Elle les considère également comme les personnes ayant le plus de compétence pour comprendre et soutenir une autre personne affectée par les brûlures.

\section{Description du programme}

\section{Clientèle}

Le groupe de soutien est mis à la disposition des membres de l'association. De dix à treize personnes se prévalent de ce service. En général, les femmes sont en plus grand nombre. La plupart des hommes qui participent aux rencontres sont des victimes de 
brûlures, alors que les femmes y assistent plutôt en tant que conjointe, mère ou amie d'une personne victime de brûlures. L'âge des participants se situe entre 20 et 65 ans. Au fil des rencontres, le nombre de couples s'accroît, alors qu'auparavant la majorité des participants venaient seuls aux rencontres.

\section{Objectifs poursuivis}

L'objectif général du groupe de soutien est d'apporter du soutien aux personnes atteintes de brûlures et à leurs proches. Les objectifs particuliers sont les suivants:

- que les membres partagent leur vécu;

- que les membres expriment leurs sentiments, leurs difficultés;

- que les membres reprennent confiance en leurs capacités personnelles et sociales ;

- que les membres, avec le soutien et l'expérience des autres membres, trouvent des solutions aux problèmes auxquels ils font face.

\section{Groupe ouvert}

Le groupe proposé par FLAM est ouvert, c'est-à-dire continuellement accessible à de nouveaux membres (Schloper et Galinsky, 1984). Les membres peuvent donc obtenir des informations et des services de façon continue. Les personnes viennent lorsqu'elles en ressentent le besoin et maintiennent leur participation tant et aussi longtemps qu'elles le désirent. Certaines se présentent à une ou deux rencontres, alors que d'autres y assistent régulièrement et constituent le noyau de base. Ainsi, les membres viennent et repartent selon leur propre rythme de développement et de changement. Comme cette formule n'exige pas d'engagement à long terme, elle peut paraître moins menaçante, exiger moins d'implication et faciliter ainsi la participation (Darveau-Fournier et Home, 1990).

Pour contrer les inconvénients occasionnés par les changements fréquents dans la composition du groupe, celui-ci s'assure d'avoir un noyau stable de participants qui transmettent les traditions du groupe, des responsables qui parrainent les nouveaux membres, des mécanismes d'intégration tels qu'un contact pré-groupe et la possibilité de s'impliquer dans une tâche particulière. 


\section{Déroulement des rencontres}

Le groupe se réunit une fois par mois. Les rencontres durent normalement deux heures, mais elles sont souvent prolongées de trente minutes. Généralement, il n'y a pas de thème prédéterminé. Si on accueille un nouveau membre, la priorité d'expression lui est accordée. Hormis cette circonstance, les sujets discutés émergent des préoccupations des membres et de leurs besoins immédiats exprimés au début des rencontres. Les échanges se poursuivent également lors des pauses, au moment où un goûter est servi. Cela facilite les contacts et favorise l'établissement d'un climat de confiance préalable à l'établissement du soutien et de l'entraide (Shulman, 1979). Cependant, au cours de la dernière année, à la demande des participants, les réunions mensuelles du groupe se sont enrichies d'un volet éducation. Des conférenciers ont renseigné les membres sur divers sujets, tels la confection, l'usage et l'entretien des vêtements compressifs, les similitudes chez les personnes entre la perte d'un être cher et celle de l'identité, le traitement des brûlures, la culture cutanée, les services offerts en ergothérapie et en physiothérapie ainsi que les bienfaits de l'entraide.

Le climat doit être sécurisant et non blâmant pour le partage entre les membres. De temps à autre, des membres rappellent le code d'éthique qu'ils se sont donné: «Je parle de moi, de ce que je ressens, de ce que je vis. Je suis à l'écoute de moi, je ne suis pas seul(e) à avoir quelque chose à dire... l'autre en a autant que moi... Je laisse la personne qui s'exprime finir ses propos; je l'interromps seulement en cas de nécessité. J'ai le respect de moi-même et aussi le respect des autres. Je ne me juge ni ne juge jamais les autres.» Vers la fin de la soirée, chaque participant peut, s'il le désire, exprimer son opinion sur le contenu de la rencontre et évaluer le bénéfice qu'il en retire. L'accolade termine la rencontre officiellement.

\section{Rôle de l'intervenante}

L'association des grands brûlés a requis les services d'une intervenante pour animer le groupe. Formée en travail social, sa principale fonction consiste à suppléer aux ressources des membres du groupe. Il lui arrive à l'occasion d'agir à titre de consultante ou de personne-ressource en donnant des avis, des conseils adéquats et en complétant l'information par des connaissances spécialisées. Pour respecter la philosophie de l'entraide, elle doit 
limiter ses interventions aux moments où le groupe requiert un renseignement pertinent ou lorsqu'une situation peut devenir nuisible à une personne ou à l'ensemble du groupe (Hopmeyer, 1990). Elle limite ses interventions non pas parce qu'elle refuse de partager ses connaissances et ses compétences professionnelles, mais parce qu'elle favorise le plus d'autonomie possible chez les membres. En fait, pour que les membres puissent s'aider mutuellement, l'accent doit être placé principalement sur leurs ressources et non sur les connaissances de l'intervenante. Aussi celle-ci reconnaît-elle la valeur de la connaissance acquise par l'expérience vécue par les membres et l'aide apportée par les pairs. Enfin, elle reconnaît aussi qu'elle ne peut comprendre toutes les facettes des expériences vécues par les membres. Si elle se sent mal à l'aise du fait qu'elle n'a pas expérimenté les situations vécues par des membres, elle partage son malaise. Elle apporte donc une assistance, mais respecte les membres en tant que preneurs de décision. L'intervenante partage les sentiments de solidarité, les valeurs et les objectifs du groupe, afin de guider les membres dans un processus de groupe. Son rôle demeure néanmoins actif au sein du groupe de soutien, alors qu'elle favorise les échanges entre les participants, les aide à exprimer ou à clarifier leurs sentiments, leurs émotions, leur vécu.

Il est important de souligner que la place occupée par l'intervenante au sein des rencontres est très variable. Etant au service du groupe et désirant lui laisser le plus d'initiatives possible, l'intervenante occupe un rôle oscillant de la position centrale à la position périphérique, et ce, parfois dans une même rencontre. C'est le besoin du groupe qui détermine le degré de participation de l'intervenante.

L'intervenante est également appelée à remplir des fonctions connexes, telles que vérifier l'aptitude d'adhésion d'un membre au groupe de soutien et le préparer. Habituellement, cette préparation s'effectue au cours d'une rencontre préliminaire. Elle vise à faire connaître les règles de fonctionnement du groupe, sa philosophie et ses objectifs. Cette préparation contribue à diminuer l'anxiété du futur participant. Celui-ci se sent plus en confiance, sachant qu'il côtoiera des personnes ayant vécu des expériences similaires. Par ailleurs, lorsque l'inter venante évalue que la personne n'est pas apte à se joindre à un groupe de soutien, elle la dirige vers une ressource mieux adaptée à sa condition. 


\section{Résultats}

À la lumière des témoignages, il est possible d'avancer que le groupe permet surtout de répondre au besoin d'échange des participants. Certains trouvent des réponses à leurs questions. D'autres y puisent du soutien afin de continuer à accompagner une personne victime de brûlures. Le fait de participer au groupe de soutien ne réduit pas la gravité des difficultés liées à leur situation particulière de vie, mais contribue à alléger le fardeau émotionnel qui lui est associé.

Des membres estiment avoir acquis des habiletés, jusqu'alors inconnues, telles que s'adresser à un auditoire, occuper un poste au sein d'un conseil d'administration. Certains établissent des liens entre eux en échangeant leur numéro de téléphone, leur adresse. Les uns offrent l'hospitalité aux personnes de l'extérieur de la région qui viennent à Québec pour un rendezvous avec un spécialiste de la santé. D'autres apportent soutien et réconfort aux membres affectés par les épreuves, telles que la maladie, une rupture.

Le groupe est aussi une occasion de s'ouvrir à la communauté, de devenir aidant à son tour, une fois résorbée sa propre situation de crise. Ainsi, des personnes atteintes de brûlures ou des proches acceptent de parrainer un individu ou une famille en situation de besoin à la suite d'un traumatisme causé par une brûlure. D'autres acceptent de rencontrer des parents désemparés qui ne savent ni quoi dire ni quoi faire pour convaincre leur enfant de ne plus jouer avec le feu. Certains participent à des activités de sensibilisation, d'éducation ou de prévention dans les écoles, afin de favoriser la réinsertion dans son milieu scolaire d'un enfant atteint de brûlures.

Quant à la présentation des thèmes particuliers, les participants ont apprécié la pertinence des sujets abordés, mais ils ont exprimé le souhait qu'un temps de partage leur soit accordé dans ce type de rencontres pour discuter de leurs besoins immédiats.

\section{CONCLUSION}

Le groupe de soutien mis sur pied par l'Association des grands brûlés FLAM est une composante essentielle du programme offert à ses membres. Il constitue un environnement propice au développement de l'aide mutuelle. Il en ressort ainsi une 
expérience stimulante pour toutes ces personnes en brisant leur isolement, en favorisant leur prise en charge et, subséquemment, en facilitant leur réinsertion sociale.

Devant cet événement extrêmement traumatisant, les personnes se culpabilisent, se sentent démunies et craignent d'être rejetées. Il est alors important que toutes les victimes de brûlures et leurs proches puissent profiter d'une expérience semblable à la nôtre. Ces personnes ont besoin d'être comprises, de normaliser leur vécu et de s'épanouir à tous points de vue. La présence d'un réseau de soutien social et l'aide professionnelle viennent faciliter cette démarche.

\section{Références bibliographiques}

BurG, G. (1990). «Témoignage», Soins, n 575/576, p. 27-28.

DARVEAU-Fournier, L., et A. Home (1990). «Le groupe au service des familles / Analyse comparative de deux types d'interventions complémentaires ", Service social, vol. 39, no 1, p. 75-96.

GAGNON, D. (1994). "Grand brûlé, une vie parsemée d'embûches », Le Soleil, 23 avril, p. A3.

GARDNEY HARLEY, N. (1981). "Treating a patient includes treating the family », dans M.M. Wagner, Care of the burned-injured patient: A multi-disciplinary involvement, London: Croom Helm.

Giguère, D. (1988). Aimez-moi, Lac-Etchemin : Comunik inc.

HOME, A., et L. DARVEAU-Fournier (1980). «La spécificité du service social des groupes », Service social, vol. 29, n ${ }^{\circ} 1-2$, p. 16-31.

Hopmeyer, E. (1990). «Entraide et service social des groupes», Service social, vol. $39, \mathrm{n}^{\circ} 1, \mathrm{p} .64-74$.

KNUDSON-COOPER, M.S. (1981). «Adjustment to visible stigma: the case of the severely burned», Social science and medicine, vol. 15b, p. 31-44.

MANNON, J.M. (1985). Caring for the burned: Life and death in a hospital burn center, Springfield, Ill. : Thomas.

MiNistère DE LA SANTÉ NATIONALE ET DU BIEN-ÊTRE SOCIAL - CANADA (1985). Unité de soins aux brûlés / Directives pour l'établissement de normes régissant les unités/programmes dans les institutions, Ottawa: Santé et Bien-être social Canada.

Munster, A.M. (1993). Severe burns / A family guide to medical and emotional recovery, Baltimore, MD: The Johns Hopkins University Press. 
NoËL, C. (1991). "Devenir social et réinsertion des brûlés », Réadaptation, $\mathrm{n}^{\circ} 376$, p. 24-27.

PAPELL, C. et B. RothMAN (1966). "Social group work model : possession and heritage », Journal of education for social work, vol. 2, $\mathrm{n}^{\circ} 2$, p. 66-77.

QUATTRINA, R. (1993). "Rôle du service social dans un centre de grands brûlés », Soins, n ${ }^{\circ}$ 575/576, p. 52-53.

RENAUD, C., et L. TESSIER (1993). Du phénomène de l'entraide au processus d'aide mutuelle en service social des groupes, Québec: Université Laval, École de service social, Faculté des sciences sociales.

SCHOLPER, J., et M.J. GALINSKY (1984). «Meeting practice needs : conceptualizing the open-ended group » Social work with groups, vol. 7, $\mathrm{n}^{\circ} 2$, p. 3-21.

SHUlman, L. (1979). The skills of helping individuals and groups, Itasca, Ill. : F.E. Peacook Publishers.

Statistique CANADA. La morbidité hospitalière, Ottawa: Statistique Canada, 82-216.

TurCotTE, D. (1993). Le soutien social, texte inédit.

Villeneuve, T. (1987). "Le grand brûlé : processus de deuil et réadaptation ", Intervention, $\mathrm{n}^{\circ} 77$, p. 52-61.

WEINBERG, N., et N.J. MiLler (1983). «Burn care : a social work perspective», Health and social work, vol. 8, n² 2, p. 97-105. 